Computing 8, 200-201 (1971)

C by Springer-Verlag 1971

\title{
Berichtigung zu Algorithmus 10
}

Von

H. Späth, Nürnberg

(Eingegangen am 4. März 1971)

Die Prozedur [1] rechnet nur für $\Delta x_{i}=\Delta y_{j}=\mathrm{I}(i=1, \ldots, n-1 / j=$ $=1, \ldots, m-1$ ) richtig. Im Allgemeinfall korrekt funktioniert das Programm, wenn folgende Änderungen vorgenommen werden. (Die Seitenzahlen beziehen sich auf [1].)

1. Die Felder $d x 2$ und $d y 2$ brauchen nicht definiert zu werden; der Parameter $d x 2$ in prepare braucht dann auch nicht zu erscheinen.

2. Ersetze Zeile 12 auf Seite 180 durch

begin for $i:=1$ step 1 until $n 1$ do

begin $d x[i]:=1 /(x[i+1]-x[i])$;

end;

$d x 1[i]:=d x[i] \times d x[i]$

3. Entferne die Zeilen 18 und 19 auf Seite 180.

4. Ersetze die Zeilen 31 bis 33 auf Seite 180 durch

$$
\begin{aligned}
& +d x 1[i-1] \times(u[i, j]-u[i-1, j])) ; \\
& p[2, j]:=p[2, j]-d x[1] \times p[1, j] ; \\
& p[n 1, j]:=p[n 1, j]-d x[n 1] \times p[n, j] ;
\end{aligned}
$$

5. Ersetze die letzte Zeile auf Seite 180 und die beiden ersten Zeilen auf Seite 181 durch

$$
\begin{aligned}
& +d y 1[j-1] \times(u[i, j]-u[i, j-1])) ; \\
& q[i, 2]:=q[i, 2]-d y[1] \times q[i, 1] ; \\
& q[i, m 1]:=q[i, m 1]-d y[m 1] \times q[i, m] ;
\end{aligned}
$$

6. Ersetze die Zeile 20 auf Seite 181 durch

$$
\text { begin matrix }(d x[i], b) \text {; }
$$

7. Ersetze die Zeile 36 auf Seite 181 durch

$$
\text { matrix }(d y[j], e) \text {; }
$$

8. Ersetze in Zeile 22 auf Seite 181

$$
k \text { durch } k 1 \text {. }
$$

Die Gleichungssysteme auf Seite 179 sind dann symmetrisch, wenn die $i$-ten Zeilen durch $\Delta x_{i-1} \Delta x_{i}(i=2, \ldots, n-1)$ bzw. die $j$-ten Zeilen 
durch $\Delta y_{j-1} \Delta y_{j}(j=2, \ldots, m-1)$ dividiert werden. Das korrigierte Programm löst die Gleichungssysteme in dieser modifizierten Gestalt.

\section{Literatur}

[1] SpÄтH, H.: Algorithmus 10 - Zweidimensionale glatte Interpolation. Computing 4, 178-182 (1969).

Dr. H. Späth

IDV-Operations Research Großversandhaus Quelle Gustav Schickedanz $K G$

Wandererstraße 159

D. 85 Nürnberg

Deutschland 